\title{
Modificações Técnicas no Transplante Cardíaco Ortotópico
}

\author{
José Teles de MENDONÇA*, José WANDERLEY NETO**, Marcos Ramos CARVALHO*, Rika Kakuda \\ da COSTA*, Luís Daniel TORRES ${ }^{\star \star}$, Ricardo de Carvalho LIMA***
}

\begin{abstract}
MENDONÇA, J. T.; WANDERLEY NETO, J.; CARVALHO, M. R.; COSTA, R. K.; TORRES, L. D.; LIMA, R. C. - Modificações técnicas no transplante cardiaco ortotópico. Rev. Bras. Cir. Cardiovasc., 9 (3): 146 $151,1994$.

RESUMO: Apresentamos algumas modificaçōes na técnica convencional de implante de coração nos transplantes cardiacos ortotópicos. As modificaçōes propostas consistem basicamente em: 1) ressecar, durante a cardiectomia, o máximo do coraçāo doente deixando apenas tecido suficiente para permitir a sutura do novo coração, durante o implante; 2) abrir o átrio direito do coração doador, a partir da veia cava inferior, próximo e paralelamente ao septo interatrial até a parte mais alta, quando a incisão deve ser bruscamente direcionada para a aurícula direita; 3 ) iniciar o implante pelo tronco pulmonar e 4) suturar os átrios conjuntamente, em um só plano, ao nivel do septo. Estas modificaçōes técnicas foram utilizadas em todos os pacientes transplantados nas três Instituiçōes e apresentam como vantagens: 1) perfeito alinhamento do tronco pulmonar, evitando-se acotovelamentos e torçōes e 2) cavidades atriais menores, sem suturas salientando para dentro dos átriose, conseqüentemente, menores oportunidades de fenômenos tromboembólicos ou contraçōes assincrônicas dos átrios (doador-receptor) que, além de favorecer a formação de trombos, podem interferirna suficiência das valvas atrioventriculares. O método é facilmente reproduzivel e pode ser recomendado para os transplantes ortotópicos de coração.
\end{abstract}

DESCRITORES: transplante cardíaco, ortotópico.

\section{INTRODUÇÃO}

O transplante de coração é hoje uma modalidade terapêutica universalmente aceita como primeira opção no tratamento de pacientes com doença cardiaca em fase terminal.

Embora o primeiro transplante de coração em humanos tenha sido realizado em 1967 por Cristian BARNARD ${ }^{6}$ na cidade do Cabo-África do Sul, toda a padronização técnica já havia sido elaborada por LOWER \& SHUMWAY ${ }^{15}$, na Universidade de Stanford-EUA. De lá para cá, mais de 19.000 transplantes ortotópicos já foram realizados, utilizandose a técnica inicialmente padronizada 20 .
Com a extensão dos transplantes de coração para o tratamento de cardiopatias congênitas complexas $1,3,5,8-10,14,17,19,22,23$, bem como a introdução do procedimento chamado "transplante em dominó" 21, 26, 28, 29, algumas modificaçōes foram feitas para ajustar o implante sem, no entanto, modificar, fundamentalmente, a técnica original.

Recentemente, DREYFUS et alii ${ }^{12}$, apresentaram uma alternativa técnica, a qual denominaram "transplante cardiaco ortotópico total", que consiste na ressecção total do coração doente e implante do enxerto com suturas diretamente ao nível das veias cavas e veias pulmonares, separadamente.

Trabalho realizado em colaboraçăo entre a Unidade Cardiotorácica de Sergipe - Hospital de Clínicas Dr. Augusto Leite, a Unidade de Tratamento Cardiotorácico (UNITÓRAX) do Real Hospital Português do Recife, e o Instituto de Doenças do Coraçăo da Santa Casa de Misericórdia de Maceió, AL, Brasil.

Apresentado ao $21^{\circ}$ Congresso Nacional de Cirurgia Cardiaca. Porto Alegre, RS, 24 a 26 de março, 1994.

- Do Hospital de Clínicas Dr. Augusto Leite, Sergipe.

* Do Instituto de Doenças do Coração, Maceió.

*.. DO UNITÓRAX, Recife.

Endereço para correspondência: José Teles de Mendonça. Rua Francisco Porto, 1882, ap. 1001. CEP 49020-120 Aracajú, SE, Brasil. 
Em 1986, ainda na fase preparatória do nosso programa de transplantes de coração, introduzimos, intuitivamente, algumas modificações técnicas, que foram utilizadas posteriormente em todos os transplantes efetuados nas três Instituições.

O presente trabalho tem como objetivo apresentar essas modificações e discutir as eventuais vantagens na prática clínica.

\section{CASUÍSTICA E MÉTODOS}

\section{Técnica Cirúrgica}

A operação é conduzida segundo os parâmetros já estabelecidos quanto a anestesia, toracotomia, canulação e circulação extracorpórea. As modificações propostas estão situadas nas fases de cardiectomia, preparo do coração doador (enxerto) e implante.

Durante a cardiectomia, o coração doente deve ser amplamente ressecado, deixando-se apenas tecido atrial suficiente para permitir a sutura do novo coração durante o implante (Figura 1). No preparo do coração doador, o átrio direito deve ser aberto

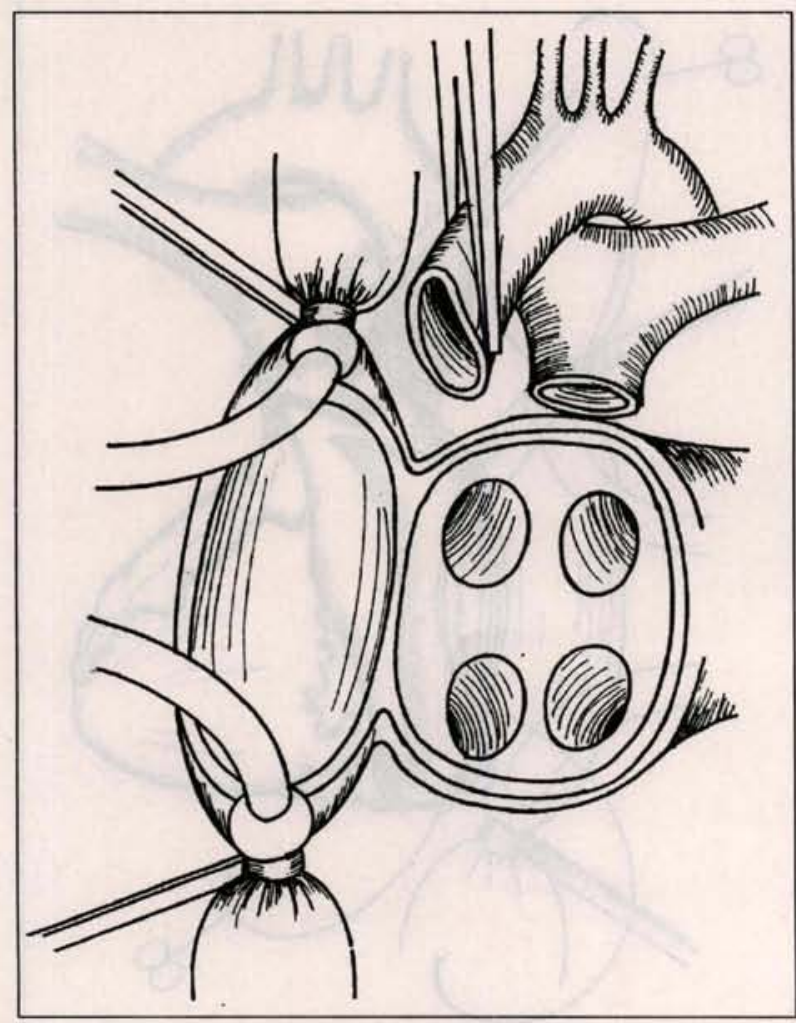

Fig. 1 - Preparo do receptor: ressecção ampla do coração doente, deixando-se apenas tecido atrial suficiente para permitir o implante.

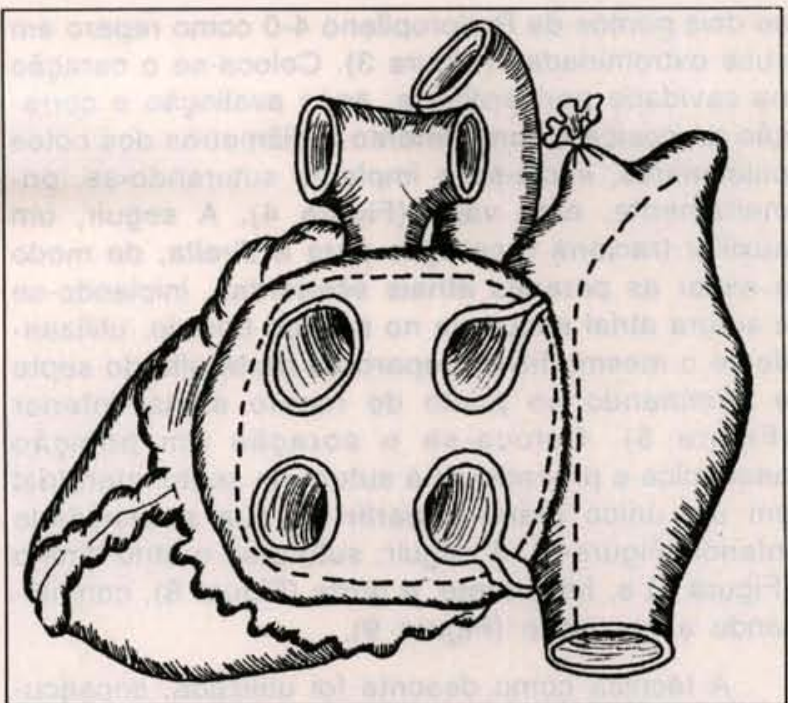

Fig. 2 - Preparo do coração doador (enxerto): as linhas tracejadas indicam o local das incisōes ao nivel dos átrios.

a partir da veia cava inferior, junto ao septo interatrial, até atingir a sua parte mais alta. Neste momento, a incisão deve mudar bruscamente de direção, dirigindo-se para a aurícula (Figura 2). O átrio esquerdo é aberto, retirando-se o tecido entre os orifícios das quatro veias pulmonares e, posteriormente, ressecando-se pequena parte de tecido atrial presa ao septo (Figura 2). Compatibilizam-se os tamanhos dos septos (doador-receptor) colocando-

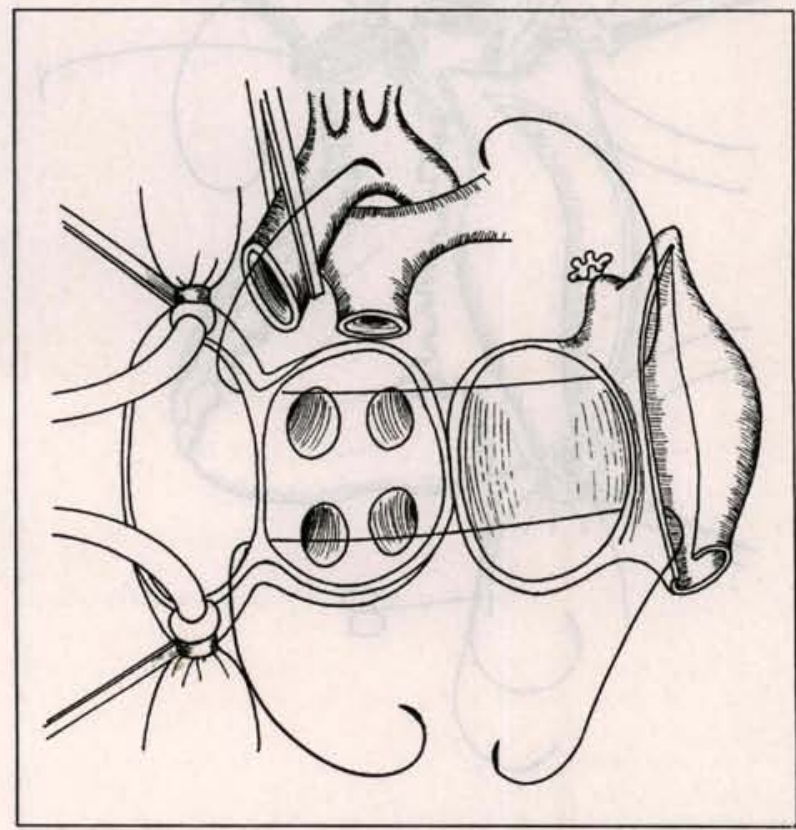

Fig. 3 - Base receptora e enxerto, colocados lado a lado, mostrando a compatibilizaçăo dos septos com dois pontos de reparo. 
se dois pontos de Polipropileno 4-0 como reparo em suas extremidades (Figura 3 ). Coloca-se o coração na cavidade pericárdica e, após avaliação e correção da posição, comprimento e diâmetros dos cotos pulmonares, inicia-se o implante suturando-se, primeiramente, este vaso (Figura 4). A seguir, um auxiliar traciona o coração para a direita, de modo a expor as paredes atriais esquerdas, iniciando-se a sutura atrial esquerda no sentido horário, utilizando-se o mesmo fio de reparo da parte alta do septo e terminando no ponto de reparo septal inferior (Figura 5). Coloca-se o coração em posição anatômica e procede-se à sutura do septo interatrial em um único plano a partir da sua extremidade inferior (Figura 6). A seguir, sutura-se 0 átrio direito (Figura 7) e, finalmente, a aorta (Figura 8), completando a operação (Figura 9).

A técnica como descrita foi utilizada, consecutivamente, em 11 pacientes operados nas três Instituições, no período compreendido entre julho de 1986 e dezembro de 1993.

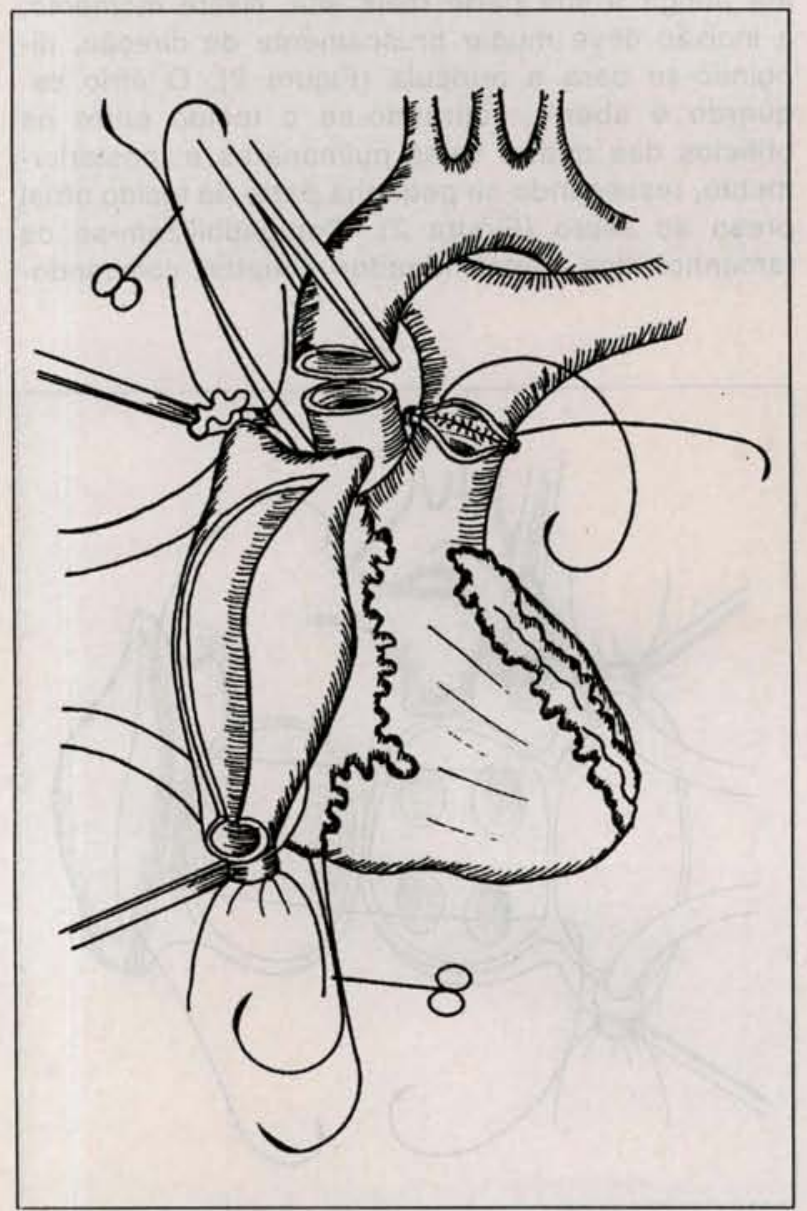

Fig. 4 - Enxerto colocado na cavidade pericárdica. Inicio das suturas pelo tronco pulmonar.

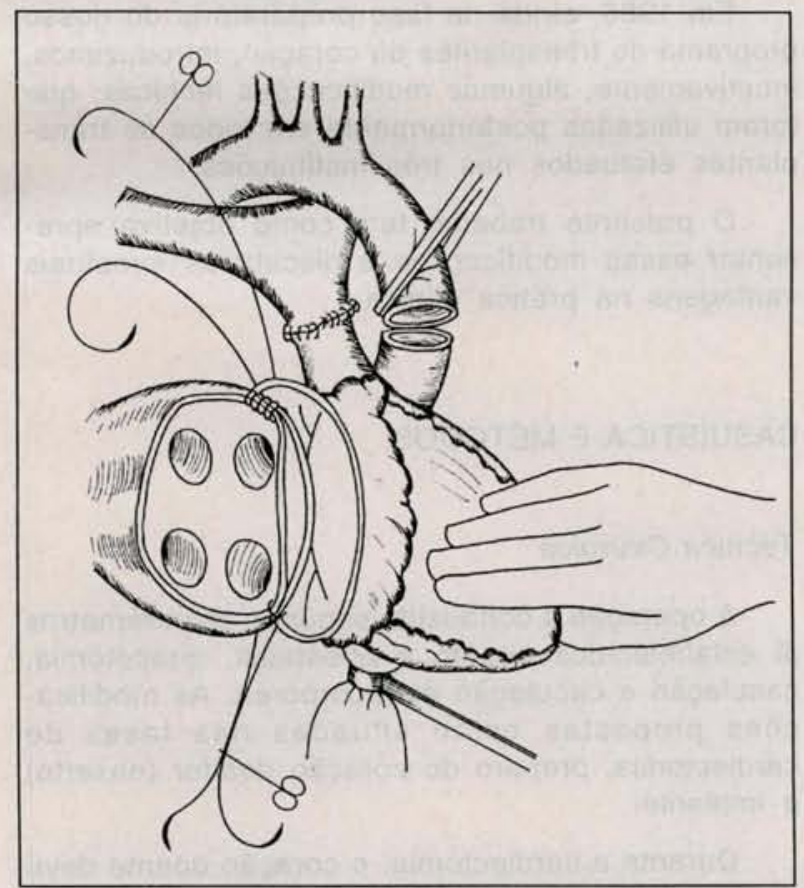

Fig. 5 - Tronco pulmonar já suturado. Auxiliar traciona o enxerto para a direita e a sutura atrial esquerda é realizada no sentido horário.

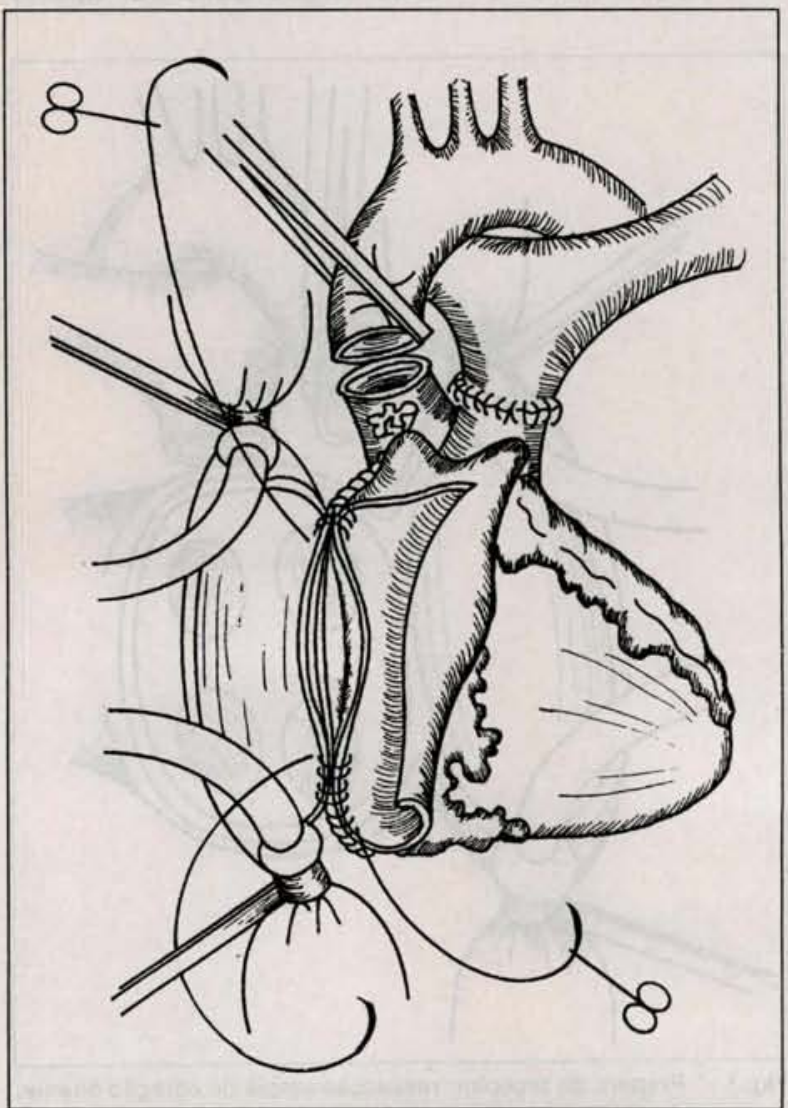

Fig. 6 - O enxerto em posição anatómica. Sutura do septo realizada em um único plano. 


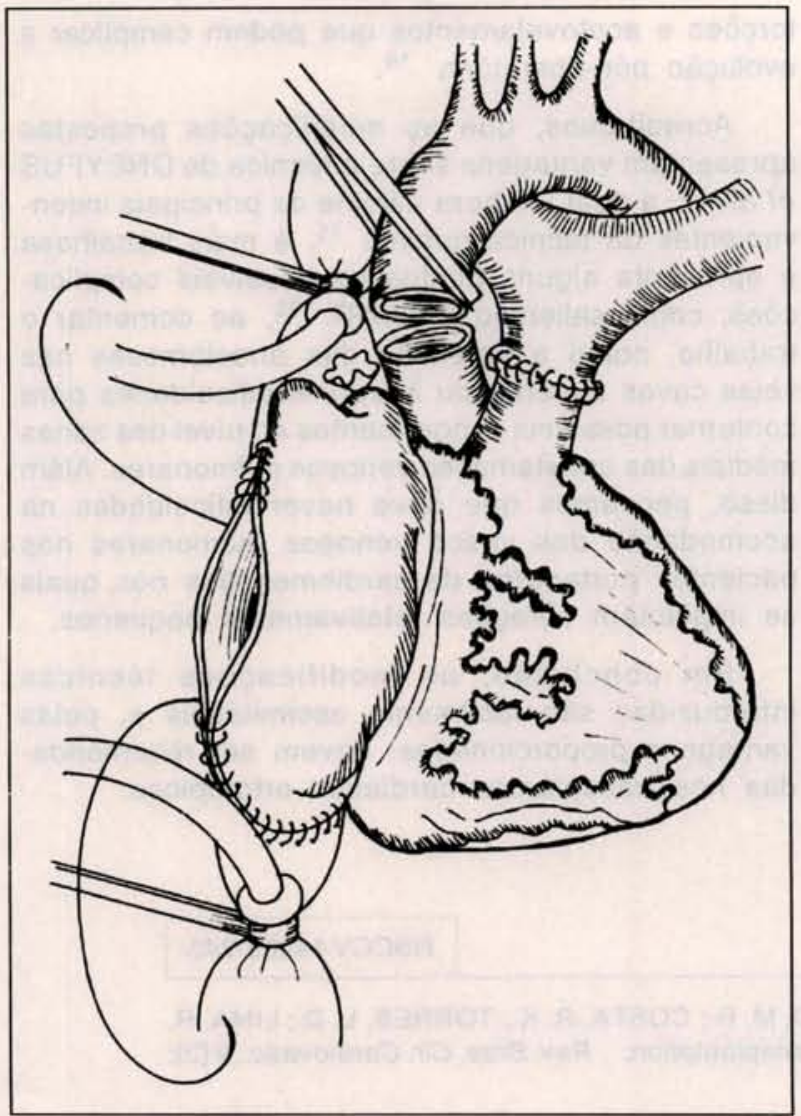

Fig. 7 - Sutura do átrio direito.

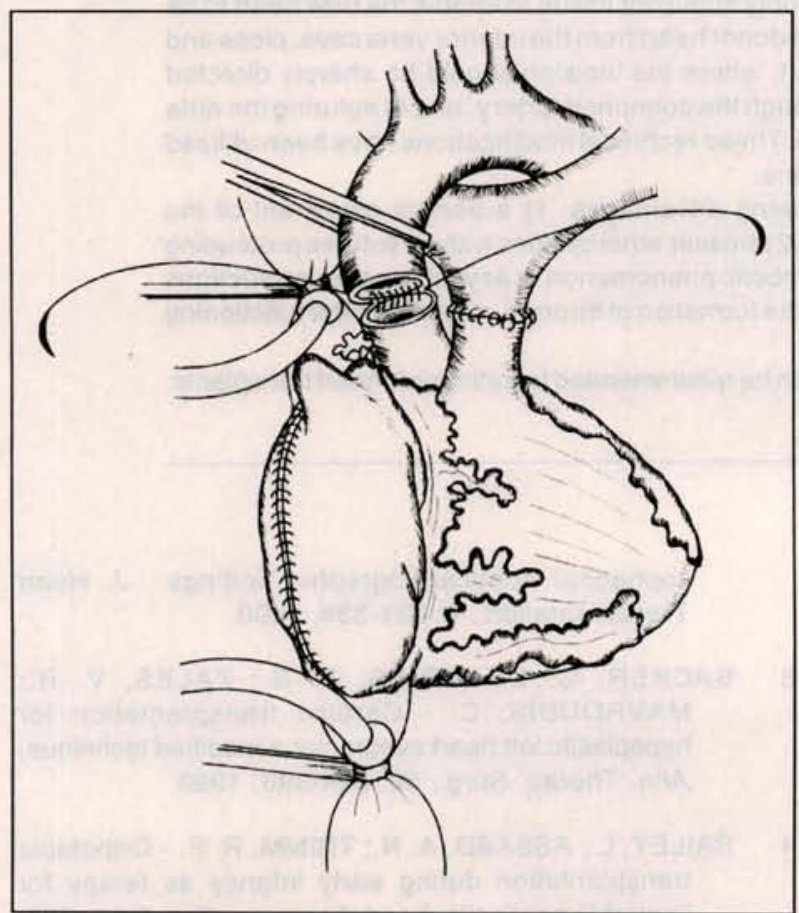

Fig. 8 - Sutura da aorta.

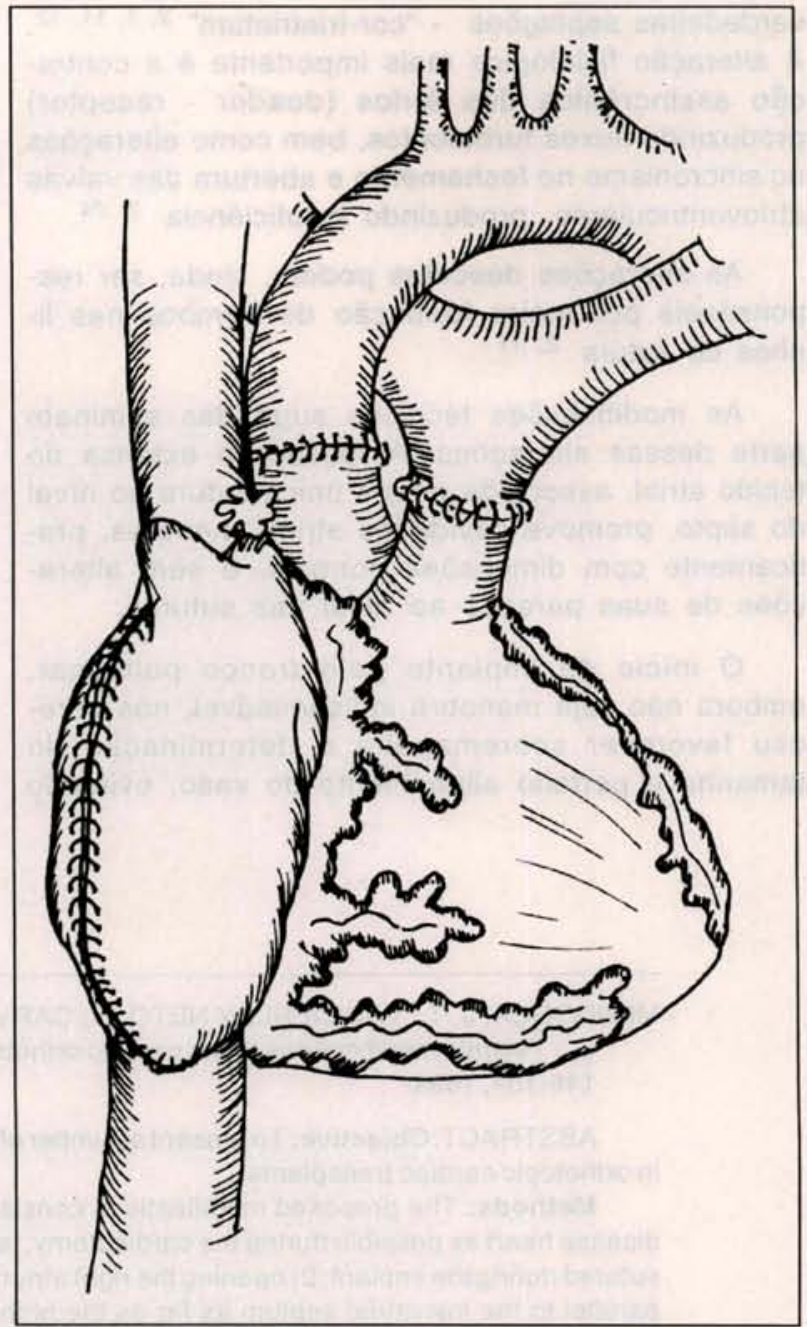

Fig. 9 - Completada a operação.

\section{COMENTÁRIOS}

Embora a técnica descrita por LOWER \& SHUMWAY ${ }^{15}$ esteja devidamente consagrada, dois pontos, no entanto, merecem algumas considerações. Em primeiro lugar, a operação, como proposta, consiste, em realidade, numa ressecção ventricular (preservando-se os átrios do receptor) e o implante de um coração novo (átrios e ventrículos). Outro problema é a sutura separada dos átrios ao nível do septo interatrial. Estes dois aspectos induzem a algumas alterações de ordem anatômica e funcional, conforme bem demonstradas com a utilização rotineira da ecodopplercardiografia e, em especial, o eco transesofágico, no acompanhamento de pacientes transplantados $2,7,12,13$. As alterações anatômicas incluem grandes átrios com linhas de sutura para dentro das cavidades, produzindo 
MENDONÇA, J. T.; WANDERLEY NETO, J.; CARVALHO, M. R.; COSTA, R. K.; TORRES, L. D.; LIMA, R. C. - Modificaçōes técnicas no transplante cardíaco ortotópico. Rev. Bras. Cir. Cardiovasc., 9(3): 146-151, 1994.

verdadeiras septações - "cor-triatriatum" 2, 7, 11, 12 . A alteração fisiológica mais importante é a contração assincrônica dos átrios (doador - receptor) produzindo fluxos turbulentos, bem como alterações no sincronismo no fechamento e abertura das valvas atrioventriculares, produzindo insuficiência $2,24$.

As alterações descritas podem, ainda, ser responsáveis por maior formação de trombos nas linhas de sutura $2,11$.

As modificações técnicas sugeridas eliminam parte dessas alterações. A ressecção extensa do tecido atrial, associada a uma única sutura ao nível do septo, promove cavidades atriais menores, praticamente com dimensões normais, e sem alterações de suas paredes ao nível das suturas.

O início do implante pelo tronco pulmonar, embora não seja manobra indispensável, nos pareceu favorecer sobremaneira a determinação do tamanho e perfeito alinhamento do vaso, evitando torções e acotovelamentos que podem complicar a evolução pós-operatória 16.

Acreditamos, que as modificações propostas apresentam vantagens sobre a técnica de DREYFUS et alii ${ }^{12}$, a qual, embora elimine os principais inconvenientes da técnica original ${ }^{15}$, é mais trabalhosa e apresenta alguns pontos de possíveis complicações, como salientou STUART ${ }^{25}$, ao comentar o trabalho, como a estenose das anastomoses nas veias cavas superior ou inferior e dificuldades para contornar possíveis sangramentos ao nível das zonas mediais das anastomoses venosas pulmonares. Além disso, pensamos que deve haver dificuldades na acomodação dos vasos venosos pulmonares nos pacientes portadores de cardiomegalias nos quais se implantam corações relativamente pequenos.

Em conclusão, as modificações técnicas introduzidas são facilmente assimiláveis e, pelas vantagens proporcionadas, devem ser recomendadas nos transplantes cardíacos ortotópicos.

\section{RBCCV 44205-243}

MENDONÇA, J. T.; WANDERLEY NETO, J.; CARVALHO, M. R.; COSTA, R. K.; TORRES, L. D.; LIMA, R. C. - Technic modifications to the cardiac orthotopic transplantation. Rev. Bras. Cir. Cardiovasc., 9 (3): 146-151, 1994.

ABSTRACT:Objective: To present a number of modifications to the conventional heart implant technique in orthotopic cardiac transplants.

Methods: The proposed modifications consist basically of the following: 1) resecting as much of the disease heart as possible during the cardiectomy, leaving only sufficient tissue to enable the new heart to be sutured during the implant; 2) opening the right atrium of the donor heart from the inferior vena cava, close and parallel to the interatrial septum as far as the highest part, where the incision should be sharply directed towards the right atrial apendix; 3 ) initiating the implant through the pulmonary artery, and 4) suturing the atria simultaneously, in a single plane, at the level of the septum. These technical modifications have been utilized in all patients undergoing transplants in the three Institutions.

Remarks: The technical changes present the following advantages: 1) a perfect alignment of the pulmonary artery, thereby avoiding bending and torsion and 2) smaller atrial cavities with no sutures protouding into the atria and, consequently, less likelihood of tromboembolic phenomenon or asynchronous contractions of the atria (donor-receiver) which, in addition to facilitating the formation of thrombi, may impair the functioning of the atrioventricular valves.

Conclusions: The method is easily reproducible and can be recommended for orthotopic heart transplants.

DESCRIPTORS: heart transplantation, orthotopic.

\section{REFERÊNCIAS BIBLIOGRÁFICAS}

1 ALLARD, M.; ASSAAD, A.; BAILEY, L.; MARCELLETI, C.; MAVROUDIS, C.; ROSE, E.; STARNES, V.; VOUHÉ, P.; YACOUB, M. - Surgical techniques in pediatric heart transplantation. J. Heart Lung Transplant., 10: 808-827, 1991.

2 ANGERMANN, C. E.; SPES, C. H.; TAMMEN, A.; STEMPFLE, A.; SCHÜLTZ, A.; KEMPES, B. M.; THEISEN, R. - Anatomic characteristics and valvular function of the heart: transthoracic versus transe- sophageal echocardiographic findings. J. Heart Transplantation., 9: 331-338, 1990.

3 BACKER, C. L.; IDRISS, F. S.; ZALES, V. R.; MAVROUDIS, C. - Cardiac transplantation for hypoplastic left heart syndrome: a modified technique. Ann. Thorac. Surg., 50: 894-898, 1990.

4 BAILEY, L.; ASSAAD, A. N.; TRIMM, R. F. - Orthotopic transplantation during early infancy as terapy for incurable congenital heart disease. Ann. Surg., 208: 279-286, 1986. 
MENDONÇA, J. T.; WANDERLEY NETO, J.; CARVALHO, M. R.; COSTA, R. K.; TORRES, L. D.; LIMA, R. C. - Modificações técnicas no transplante cardiaco ortotópico. Rev. Bras. Cir. Cardiovasc., 9(3): 146-151, 1994.

BAILEY, L.; CONCEPCION, W.; SHATTUCK, H.; HUANG, L. - Method of heart transplantation for treatment of hypoplastic left heart syndrome. J. Thorac. Cardiovasc. Surg., 92: 1-5, 1986.

BARNARD, C. N. - A human cardiac transplant: an interim report of a successful operation performed at Groote Schuur Hospital, Cape Town. S. Afr. Med. J., 41: $1271,1967$.

BJORKE, R. J.; ZIADY, G. M.; MATESIC, C.; MARRONE, G. - Early diagnosis and follow-up by echocardiography of acquired cor triatriatum after orthotopic heart transplantation. J. Heart Lung Transplant, 11: 1073-1077, 1992.

CHARTRAND, C.; GUERIN, R.; KANAGH, M.; STANLEY, P. - Pediatric heart transplantation: surgical considerations for congenital heart diseases. J. Heart Transplant., 9: 608-617, 1990.

COOPER, M. M.; FUZESI, L.; ADDONIZIO, L. J.; HSU, D. T.; SMITH, C. R.; ROSE, E. A. - Pediatric heart transplantation after operations involving the pulmonary arteries. J. Thorac. Cardiovasc. Surg., 102: 386-395, 1991.

DOTY, D. B. - Cardiac transplantation. Transplantation of the great arteries. In: Cardiac surgery (A looseleaf woorkbook and up-date service). St. Louis, MosbyYear Book, 1988. p. 24-25.

DREYFUS, G. - Total orthotopic heart transplantation: an alternative to the standard technique. Ann. Thorac. Surg., 54: 187, 1992.

DREYFUS, G.; JEBARA, V.; MIHAILEANV, S.; CARPENTIER, J. - Total orthotopic heart transplantation: an alternative to the standard technique. Ann. Thorac. Surg., 52: 1181-1184, 1991.

FAGAN Jr., L. F.; PENICK, D. R.; WILLIAMS, G. A.; LABOVITZ, A. J.; PEARSON, A. C. - Twodimensional, spectral Doppler, and color flow imaging in adults with acdquired and congenital cor triatriatum. J. Am. Soc. Echocardiogr., 4: 177-184, 1991.

14 HARJULA, A. L. J.; HEIKKILA, L. J.; NIEMINEM, M.S.; KUPARI, M.; KETO, P.; MATILA, S. P. - Heart transplantation in repaired transposition of the great arteries. Ann. Thorac. Surg., 46: 611-614, 1988.

LOWER, R. R. \& SHUMWAY, N. E. - Studies of orthotopic homotransplantation of the canine heart. Surg. Forum, 11: 18-19, 1960.

MARCHENA, E.; FUTTERMAN, L.; WOZNIAK, B. S.; MADRID, W.; MITRANI, A.; MYERBURG, R. J.; BOLOOKI, H. - Pulmonary artery torsion: a potential lethal complication after orthotopic heart transplantation. J. Heart Transplant., 8: 499-502, 1989.

17 MAYER Jr. J. E.; PERRY, S.; O'BRIEN, P.; PEREZATAYDE, A.; JONAS, R.; CASTANEDA, A.;
PARNESS, I. A. - Orthotopic heart transplantation for complex congenital heart disease. J. Thorac. Cardiovasc. Surg., 99: 484-492, 1990.

18 MENDONÇA, J. T.; WANDERLEY NETO, J.; CARVALHO, M. R.; COSTA, R. K.; TORRES, L. D. - Transplante ortotópico do coração: modificaçōes técnicas no implante. Arq. Bras. Cardiol.; 61 (Supl. 2): 137, 1993. (Resumo).

19 MENKIS, A.; MCKENZIE, F. N.; NOVICK, R. J.; KOSTUK, W. J.; PFLUGFELDER, P. W.; GOLDBACH, M.; ROSEMBERG, H. - Special considerations for heart transplantation in congenital heart disease. J. Heart. Transplant., 9: 602-607, 1990.

20 THE REGISTRY of the International Society for Heart and Lung Transplantation: nineth official report. J. Heart Lung Transplant., 11: 599-606, 1992.

21 REITZ, B. A. - Heart and lung transplantation. In: BAUMGARTNER, W. A.; REITZ, B. A.; ACHUFF, S. C. Heart and lung transplantation. Philadelphia, W. B. Saunders, 1990. p. 338-339.

22. REITZ, B. A.; JAMIESON, S. W.; GAUDIANI, V. A.; OYER, P. E.; STINSON, E. B. - Method for cardiac transplantation in corrected transposition of the great arteries. J. Cardiovasc. Surg., 23: 293-296, 1982.

23. RICHENBACHER, W. E.; KARWANDE, S. V.; SHADDY, R. E.; RENLUND, D. G.; BRISTOW, M. R.; O' CONNELL, J. B.; GAY, W. A. - Cardiac transplantation in a patient with a single ventricle and transposition of the great vessels. Ann. Thorac. Surg., 52: 871. $873,1991$.

24. STEVENSON, L. W.; DADOURIAN, B. J.; KOBASHIGAWA, J.; CHILD, J. S.; CLARK, S. H.; LAKS, H. - Mitral regurgitation after cardiac transplantation. Am. J. Cardiol., 60: 119-122, 1987.

25. STUART, W. J. - Total orthotopic heart transplantation: an alternative to the standard technique. Ann. Thorac. Surg., 52: 1181-1184, 1991.

26. VASU, M. A. - A suggested technique for harvest of the cardiac graft from the heart-lung recipient in domino heart transplant. J. Heart Transplant., 9: 729-731, 1990.

27. YACOUB, M. - Method of heart transplantation for treatment of hypoplastic left heart syndrome. $J$. Thorac. Cardiovasc. Surg., 92: 1-5, 1986.

28. YACOUB, M. H. \& BANNER, N. A. - Recent developments in lung and heart-lung transplantation. In: MORRIS, D. J. \& TILNEY, M. E. (eds.) Transplantation reviews. Philadelphia, W. B. Saunders, 1989. Vol. 3, p. 1-29.

29. YACOUB, M.; KHAGHANI, A.; ARAVOT, D. - Cardiac transplantation from live donors. J. Am. Coll. Cardiol., 11: 102, 1988 (Resumo). 